\title{
The use of graphite foams for simultaneous collection and storage of concentrated solar energy
}

\author{
Heinrich Badenhorst ${ }^{*}$, Natasha Fox and Ashraf Mutalib \\ SARChI Chair in Carbon Materials and Technology, Department of Chemical Engineering, University \\ of Pretoria, Lynnwood Road, Pretoria, 0002, South Africa
}

\begin{abstract}
Graphite foams of varying composition and density were prepared using a low cost, local pitch material and expandable graphite for use in solar energy capture. The foams have a high degree of graphitization but exhibit a fine mosaic texture. A small oxidative treatment (6\% mass loss) was necessary to fully open the foam pores. As the density is reduced a large decrease in the foam surface area was observed. Despite this, an increase in solar energy capture efficiency was measured due to increased circulation through the foam. By varying foam geometry and the concentration ratio it was demonstrated that the receiver size can be reduced by $75 \%$ at the same efficiency. The foam with the lowest density was used to test the thermal performance of a simultaneous energy capture and storage concept using a phase change material. The melting time of the phase change material is reduced by $46 \%$ whilst only reducing the energy storage density by $18 \%$. In addition it was found that the foam composite resulted in more ideal phase transition behaviour due to the elimination of incongruent melting. The composite can effectively capture, store and discharge thermal energy, at a constant temperature, without any additional requirements.
\end{abstract}

\section{Introduction}

It is widely accepted that global warming has become a pressing issue which must be addressed [1, 2]. Consequently there has been an increasing, world-wide focus on utilizing renewable energy options $[3,4]$. In this context carbon is usually viewed in a

\footnotetext{
* Corresponding author. Tel: +27 12420 4989. Fax: +27 12420 2516. E-mail: carbon@up.ac.za
} 
negative light with most industries and nations trying to reduce their "carbon footprint" [5,6]. For these reasons increasing research focus has been placed on developing carbon materials for novel renewable applications [7, 8]. Solar collectors and thermal energy storage components are two key subsystems in most solar thermal applications [9]. This work reports on the potential of using graphite foams for collecting and storing concentrated solar energy.

Many different approaches exist for concentrating solar energy [10], broadly classified as line focus or point focus. The latter is capable of achieving much higher concentration ratios and hence higher temperatures, making it attractive for a wide variety of applications. Over the past decades, extensive effort has been placed on developing suitable receivers for solar energy capture [11-13]. However, the extreme conditions present at the focal point of such systems places very stringent requirements on the material properties.

Replicating these conditions on a small scale in itself poses a significant challenge which to a certain extent limits material development. Several attempts have been made to recreate these conditions artificially using electrical lighting systems [14, 15]. These configurations do achieve very high concentration ratios of between 1,000 and 10,000 suns [16] however, their costs, both capital and operational, are very high. Furthermore, when using electrical lighting systems the electromagnetic spectrum of solar radiation incident on the earth's surface is not exactly replicated. This may lead to a reduction in the performance of the material when it is practically implemented.

Thus a simple, low cost technique for generating high concentration ratios using sunlight directly would be beneficial for initial material assessment and selection. Generally Fresnel lenses have proven difficult to use due to their rigorous tracking requirements [17]. However a simple device has been developed to track and reflect sunlight onto a Fresnel lens based concentrator. The device uses only low cost materials and is capable of concentration ratios in excess of 1,000 suns. This system was used as the platform for assessing different graphitic foams for concentrated solar applications.

Traditionally, ceramic materials are used to construct solar receivers [18] because of their ability to withstand extreme temperatures. However, ceramic materials have several drawbacks, namely low thermal conductivity and brittleness. Graphite on the other has excellent thermal conductivity and good ductility [19]. In addition, graphite 
has a very low thermal expansion coefficient making it highly resistant to thermal shock, a property which is critical in solar receiver design [20]. Graphite foams can have a low density and high surface area [21] making them ideal for flow through, heat transfer applications.

Very high thermal conductivity foams are usually produced from expensive mesophase pitch [22-25] which has lately become very difficult to obtain commercially. However, recent work has focused on producing carbon foams from alternative precursors [26] including low-cost pitch precursor and intercalated graphite [27]. The thermal diffusivity of this low density foam is $1.2 \times 10^{-4} \mathrm{~m}^{2} \cdot \mathrm{s}^{-1}$ which is very close to that of pure copper, however the density of the material is 25 times less. These properties make them ideal candidates for use in solar receiver design [28]. The first objective of this investigation is to assess the performance of pitch derived foams as heat transfer medium for use in concentrated solar designs.

Due to the highly variable nature of solar energy, effective storage is essential for supplying energy during peak demand. Next to capital cost this is arguably the biggest obstacle to wide-scale implementation of renewable energy [29]. Among the most promising options for the storage of solar thermal energy are phase change materials (PCMs). Due to the utilization of latent heat rather than sensible these materials have a very high energy density. During the transition from solid to liquid energy is stored and then recovered during the reverse transformation. However, the low thermal conductivity of these materials is a significant issue [30,31]. To overcome this limitation recent research has increasingly focused on developing composites with graphite foams, to improve the thermal performance of the PCMs [32-35].

The second objective of this work is to evaluate if the graphite foams under consideration can significantly improve the heat transfer of PCMs while maintaining their energy storage capacity. However, the approach is unique in the sense that capture and storage is combined in a single step. Conventionally, solar energy is collected using a heat transfer fluid which is then used to transport the energy to the storage medium [10]. This approach has several disadvantages including the associated heat losses and the cost of the secondary materials. In this work the capture is achieved by the foam with direct distribution into the PCM for storage.

Graphite foams of varying composition and density were prepared using a locally produced pitch material. These were assessed using the solar platform mentioned 
earlier in combination with a water based calorimeter. This procedure enables rapid screening and comparison of the different materials. The most attractive material was then selected for testing the thermal performance enhancement of a PCM. Myristic acid was chosen as PCM due to its suitability for water heating applications [36]. The chosen foam was impregnated with PCM and directly heated using the solar concentrator. The time required for complete melting of the PCM is compared to that of the pure PCM. The composite has the potential to effectively capture and store thermal energy without any additional requirements. This system represents a departure from past notions on energy storage and may lead to a new paradigm in securing the continuous availability of renewable energy. An additional requirement for a practical system however is the discharge of the stored energy. This may be achieved through direct transfer from the storage container walls or by incorporation of pipes for heat exchange. The latter would decrease the energy storage density, however for this preliminary investigation into capture efficiency, discharge considerations were not taken into account.

\section{Experimental}

\subsection{Solar concentration platform}

A small scale prototype of a novel solar concentrator has been designed and fabricated for testing. The device uses a circular Fresnel lens which traditionally requires very accurate tracking to maintain its focal point. In addition, the lens requires a collimated light source, making the use of conventional electrical lighting impossible. For most practical purposes sunlight can be considered collimated making it ideal. The use of sunlight has the additional benefit of replicating the electromagnetic spectrum exactly, instead of the approximation afforded by for example metal halide lamps. Unfortunately due to the trajectory of the sun across the sky, even if the lens tracking is impeccable the focal point will not be horizontal and its position in space will move as the sun moves. For these reasons the opposite approach was adopted: the lens is kept fixed and instead sunlight is reflected through a series of mirrors, shown schematically in Figure 1. 


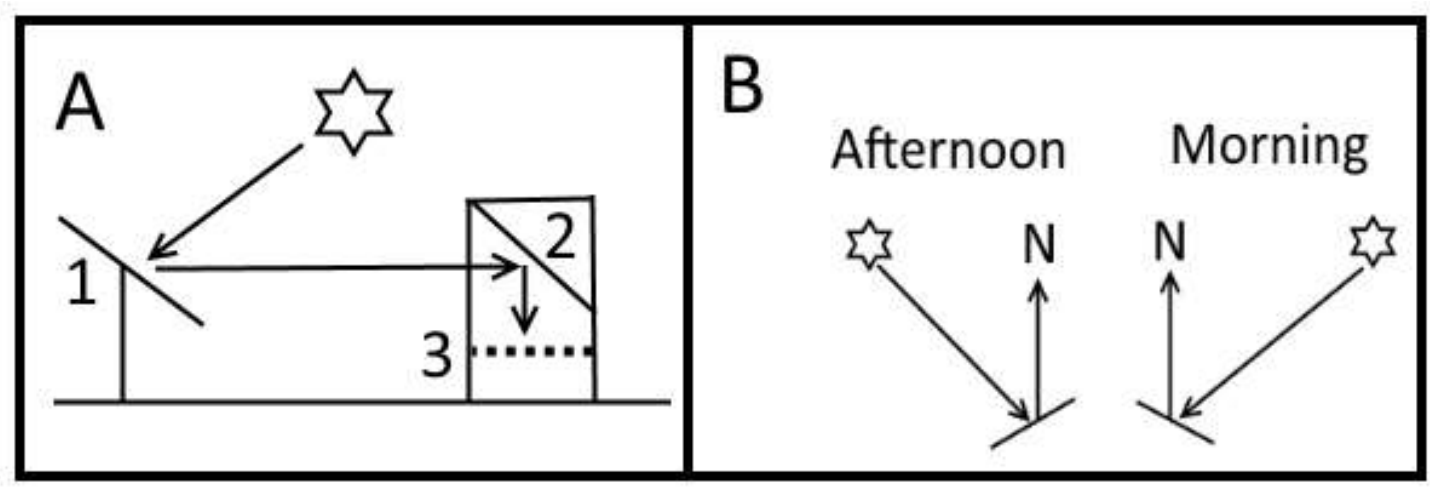

Fig. 1: Schematic of tracking system (A) side-on and (B) from above.

The platform is oriented in a North-South direction (facing North given the current location in the southern hemisphere) with mirror 1 tracking the sun and mirror 2 being stationary. As can be seen from Figure 1 B, when viewed from above, mirror 1 maintains an angle perpendicular to the direction given by the centreline of the angle between the sun and true North. In this way sunlight is reflected in a northerly direction throughout the day independent of the azimuth angle of the sun. The elevation of the sun is compensated for by adjusting the angle of the mirror with respect to the earth's horizontal level. Thus the system uses a simple two axis tracking method which has been employed extensively in photo-voltaic implementations.

The secondary mirror is not required if a horizontal beam of light can be used. However, for this investigation a vertical beam of light is preferential for the experimental setup. In this manner a downward directed, beam of collimated sunlight is produced, perfect for use with a Fresnel lens positioned at the dotted line in Figure 1, i.e. location 3 . The height of the focal point can be adjusted by varying the height of the lens. The system is shown in operation in Figure 2. 


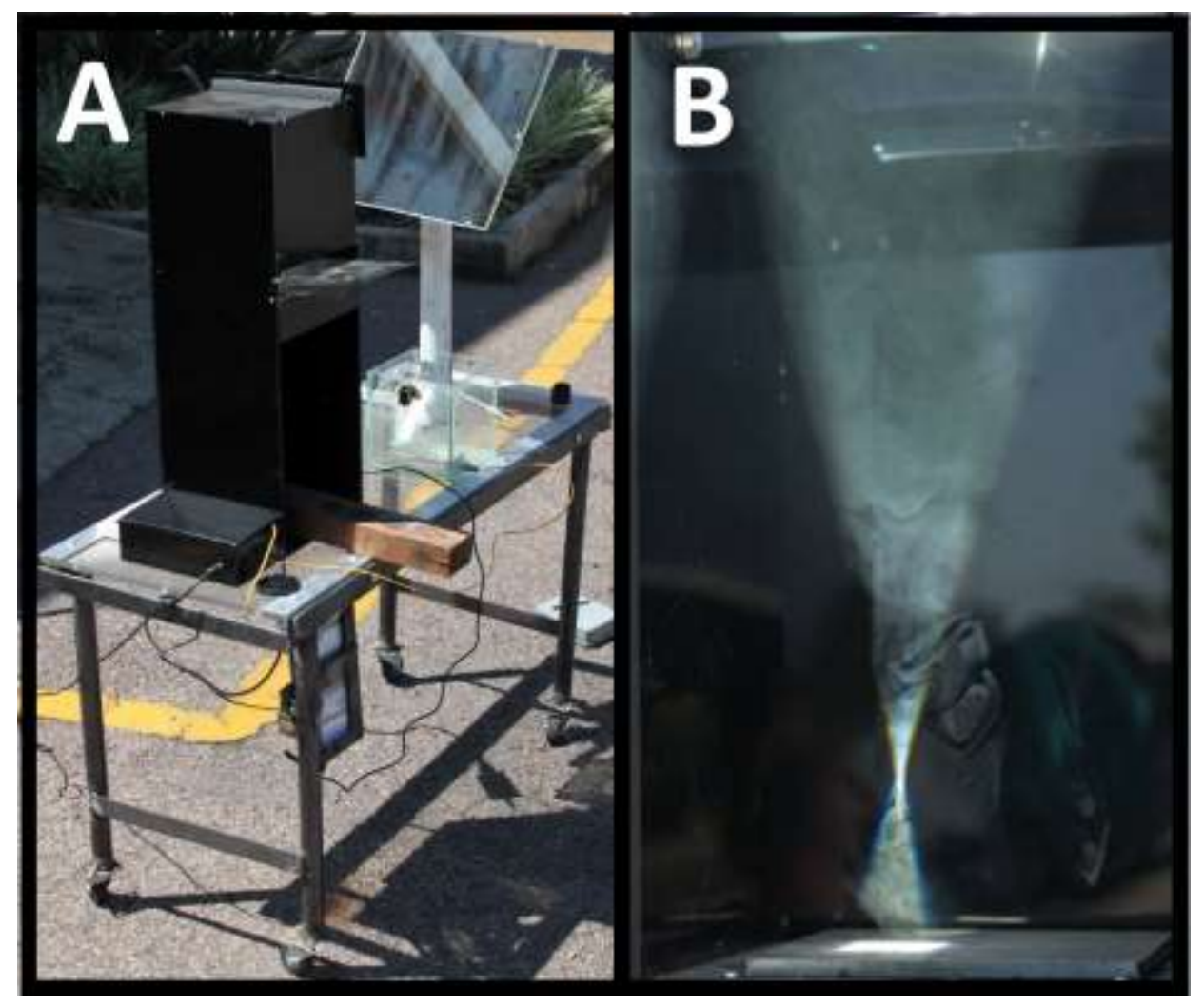

Fig. 2: (A) Operational solar concentrator and (B) focal point

As can be seen from Figure $2 \mathrm{~B}$ the assembly produces a concentrated beam of sunlight with a clear focal point. The lens is made from a polymer (PMMA) and results in a small spectral loss, as demonstrated in Figure 3, where the solar spectrum above and below the lens is compared.

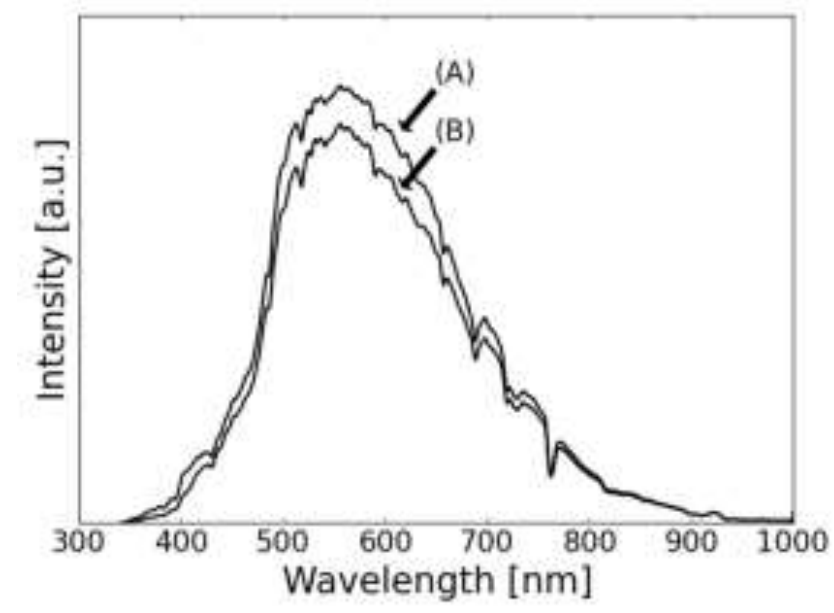

Fig. 3: Solar spectrum comparison (A) above and (B) below the lens 
An Apogee Instruments PS-100 spectro-radiometer with a spectral range of 350 to $1150 \mathrm{~nm}$ and spectral resolution of $1 \mathrm{~nm}$ was used to measure the electromagnetic spectra. In addition the spectro-radiometer is calibrated to measure the total incident solar radiation in $\mathrm{W} . \mathrm{m}^{-2}$, thus the total energy incident on a test sample can be accurately calculated. The lens leads to a reduction of $24 \%$ in the transmitted solar energy.

\subsection{Graphite foam preparation}

Coal tar pitch (110MP) was obtained from Arcelor-Mittal (South Africa) with a softening point of $63{ }^{\circ} \mathrm{C}$ and a coke yield of $\sim 44 \mathrm{wt} \%$. The quinoline insolubles (QI) and toluene insolubles (TI) are 9 and $34 \mathrm{wt} \%$, respectively. The Aromaticity Index is 0.53 and the $\mathrm{C} / \mathrm{H}$ atomic ratio is 1.83 . The expandable graphite (EG) grade ES170 $300 \mathrm{~A}$ (onset temperature $300{ }^{\circ} \mathrm{C}$ ) was obtained from Qingdao Kropfmuehl Graphite (China). According to the manufacturer, it produces $>170 \mathrm{~mL} / \mathrm{g}$ exfoliated material when heated above $300{ }^{\circ} \mathrm{C}$. This implies that a gram of material expands to fill a volume of $170 \mathrm{~mL}$ upon heating, achieving a bulk density of $\left(0.006 \mathrm{~g} . \mathrm{cm}^{-3}\right)$. The details of the foam production process are given elsewhere [24], but in short the procedure is as follows:

- Pitch is heat treated at $437^{\circ} \mathrm{C}$ for 6 hours to produce mesophase pitch

- The pitch is ground and dry mixed with expandable graphite

- The mixture is foamed in air at $460{ }^{\circ} \mathrm{C}$ and cross linked at $250{ }^{\circ} \mathrm{C}$

- The foams are then carbonized $\left(1200^{\circ} \mathrm{C}\right)$ and graphitized $\left(2600{ }^{\circ} \mathrm{C}\right)$

By changing the wt $\%$ of EG foams of varying densities are obtained. The wt $\%$ of EG was adjusted from 3 to $6 \mathrm{wt} \%$ in increments of $1 \%$. The upper limit is determined by the foam structural integrity, above this loading the foam disintegrates due to excessive expansion of the EG. The lower limit is determined by porosity, below this loading a solid pitch artefact is obtained with almost no porosity. The surface areas of the foams were determined with a Micromeritics Tristar II instrument. The bulk density was calculated and the skeletal density was measured using a Micromeritics AccuPyc II 1340 gas pycnometer. To confirm the extent of graphitization the foams were characterized using a PANalytical X-pert Pro powder diffractometer (XRD) with variable divergence and receiving slits coupled to an 
$\mathrm{X}$ 'celerator detector using iron-filtered cobalt $\mathrm{K} \alpha$ radiation. In addition the samples were visually inspected using polarized light microscopy.

\subsection{Solar testing}

To test the heat transfer capability of the foams a simple, water based calorimeter was constructed. The device is built from extruded polystyrene foam (ISOboard, South Africa). Neither molten PCM nor water is capable of penetrating the closed cell structure of the polystyrene foam. This material was chosen due to its particularly low thermal conductivity $\sim 0.024 \mathrm{~W} \cdot \mathrm{m}^{-1} \cdot \mathrm{K}^{-1}$, which keeps heat losses to a minimum. Structurally it is simply a rectangle of polystyrene foam $(13 \mathrm{~cm} \times 13 \mathrm{~cm} \times 8 \mathrm{~cm})$ with a small square cavity $(3 \mathrm{~cm} \times 3 \mathrm{~cm} \times 3 \mathrm{~cm})$ in the centre of its top surface. The top surface is covered with a thin square of double pane glass ( $3 \mathrm{~mm}$ thickness). For this preliminary work standard window glass was used which results in addition spectral losses as can be seen from Figure 4.

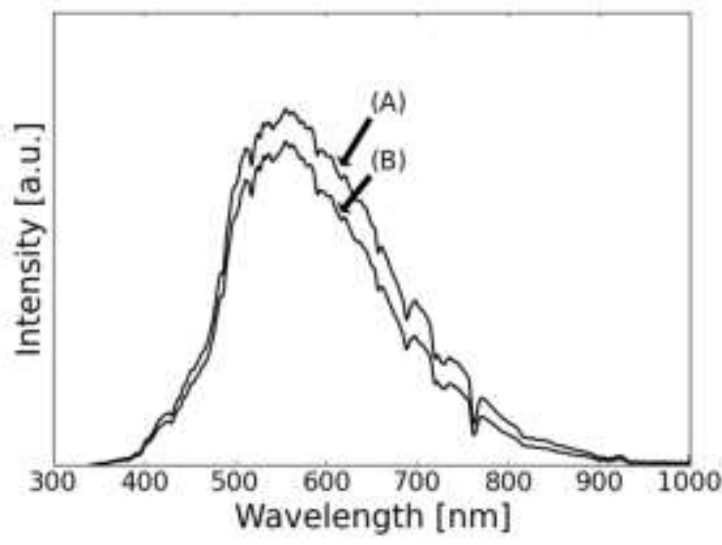

Fig. 4: Solar spectrum comparison (A) above and (B) below the glass

The glass leads to an additional reduction of $22 \%$ in the transmitted solar energy, thus a total loss of $40 \%$ is incurred for the entire path. Despite this loss in incident radiation the glass window is needed to eliminate any heat losses through water evaporation and convection at the top surface. For comparative purposes the path loss is immaterial as long as it remains constant. The cavity is filled with water and the foam is submerged in the water. After allowing the water to penetrate the foam structure the glass window is held in place with rubber bands and it is ensured that no air gaps are present. The volume of the cavity is fixed and the volume of water is determined by subtracting the foam skeletal volume. The temperature of the water is 
measured before and after the foam sample is exposed to concentrated sunlight for a fixed period of time. Care was taken to ensure that the water is thoroughly mixed when the water temperature is measured. This ensures that a representative result is obtained for the average heat transfer rate to the water during the test.

For the PCM measurements a polystyrene foam container of the same dimensions is used but in this case the cavity is entirely filled either with PCM or the PCM/foam composite. For this experiment the temperature at the bottom of the sample (i.e. the bottom of the polystyrene cavity) is continuously measured using a LabVIEW system. The myristic acid used in these experiments was obtained from Sigma-Aldrich ( $>95 \%$ purity). The PCM/foam composite was produced by impregnating the graphite foam with molten PCM under vacuum. The enthalpy of fusion of the PCM was measured using a Perkin-Elmer Differential Scanning Calorimeter (DSC 4000).

\section{Results}

The graphitic nature of the foam is easily confirmed from the XRD diffractogram shown below in Figure 5.
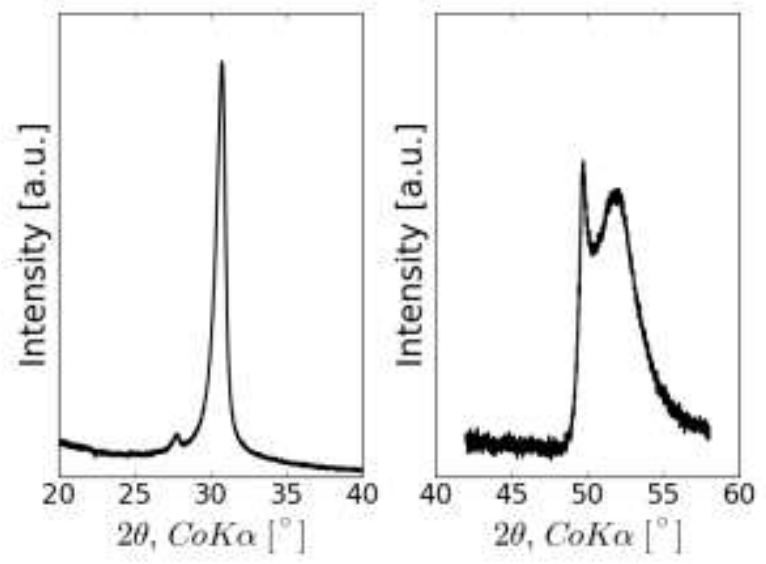

Fig. 5: XRD diffractogram of selected regions for graphitized foam

The graphitized foam clearly shows the characteristic $d_{002}$ graphite reflection at $30.9^{\circ}(C o K \alpha)$ along with the (100) and (110) peaks at around $50^{\circ}$. Whilst this confirms the presence of graphite it indicates neither the size nor the extent of alignment of the graphitic subdomains. When the foam ligaments are examined closely under polarized light however, the graphite microstructure may be observed in Figure 6. 


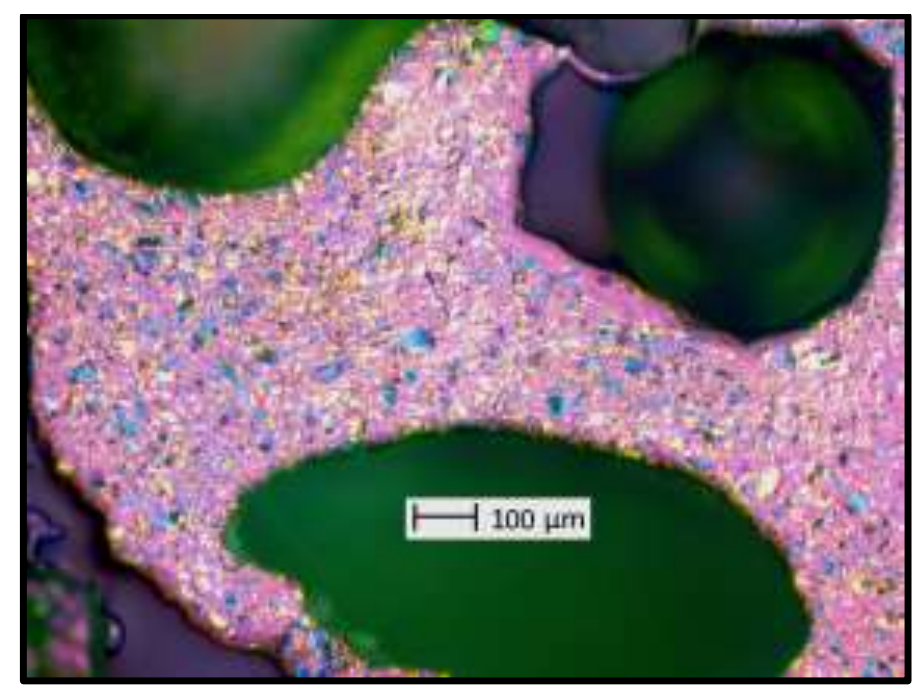

Fig. 6: Optical micrograph of foam under polarized light at 50x magnification

The sub-domains are very small and randomly oriented resulting in a fine mosaic texture. This is most likely due to the pitch milling step. Some alignment is visible near the edges of the foam ligaments but this effect is limited. This result explains the foams' fairly low thermal conductivity of around $21 \mathrm{~W} \cdot \mathrm{m}^{-1} \cdot \mathrm{K}^{-1}$ [24]. Initially however, skeletal density measurements were found to be on the order of $\sim 1 \mathrm{~g} . \mathrm{cm}^{-3}$. This is significantly lower than the theoretically expected graphite density of 2.26 g. $\mathrm{cm}^{-3}$. Thus it was concluded that the foams may have a significant portion of closed porosity. To overcome this issue a small sample was progressively oxidized in air and the results are shown below in Figure 7.

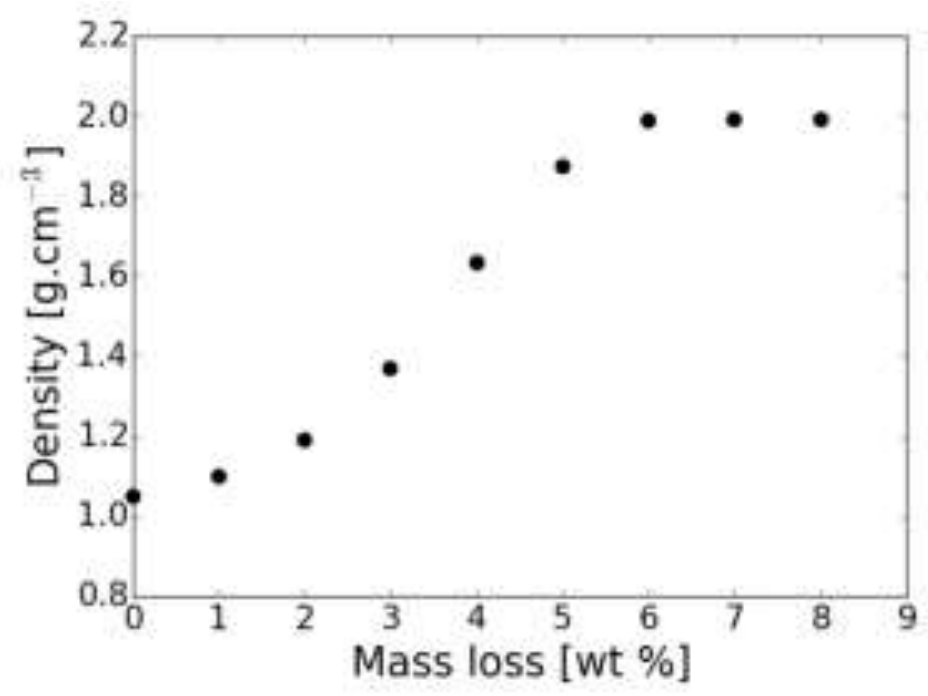

Fig. 7: Foam skeletal density as a function of oxidation 
There is a steady increase in the skeletal density as the sample is oxidized and the pores are opened up. However, the value levels off beyond $6 \%$ mass loss, indicating that all pores have been opened and additional oxidation may erode the pore walls and degrade the foam structure. The final achieved density is around $2.00 \mathrm{~g} . \mathrm{cm}^{-3}$, which corresponds to the skeletal density of the original sample measured when it is ground into a powder. The value is slightly lower than the theoretical value for a single crystal of graphite. This is in line with expectations since the microstructure of the material has a very fine mosaic texture indicating millions of small crystals with many grain boundaries and defects. In addition, given the maximum heat treatment is only $2600^{\circ} \mathrm{C}$, the transformation to graphite may not be complete.

Based on the oxidation results all foams were oxidized to a mass loss of around $6 \%$ before any heat transfer testing was done. This ensures maximum permeability of the fluid and maximum surface area for heat exchange.

To compare the overall foam performance the efficiency $(\eta)$ is calculated as:

$$
\eta=\frac{m_{W}[\mathrm{~kg}] * C_{P}[\mathrm{~J} / \mathrm{kg} \cdot \mathrm{K}] * \Delta T\left[{ }^{\circ} \mathrm{C}\right] / t[\mathrm{~s}]}{\text { incident radiation }\left[W / \mathrm{m}^{2}\right] * \text { lens area }\left[\mathrm{m}^{2}\right] * \text { loss } \%}
$$

Where $m_{W}$ is the mass of water, $C_{P}$ is heat capacity of water, $\Delta T$ is the change in water temperature from the run start to finish and $t$ is the run time. The achieved foam bulk densities as the amount of EG is increased are given in Table 1.

Table 1: Foam bulk density and surface area

\begin{tabular}{|c|c|c|}
\hline $\begin{array}{c}\text { Expandable graphite } \\
{[\mathbf{w t} \text { \%] }}\end{array}$ & $\begin{array}{c}\text { Bulk density } \\
{\left[\mathbf{g . c m}^{-\mathbf{3}}\right]}\end{array}$ & $\begin{array}{c}\text { BET surface area } \\
{\left[\mathbf{m}^{\mathbf{2}} \mathbf{. g}\right.}\end{array}$ \\
\hline 3 & 0.419 & 4.13 \\
\hline 4 & 0.384 & 3.14 \\
\hline 5 & 0.355 & 2.04 \\
\hline 6 & 0.299 & 1.40 \\
\hline
\end{tabular}

As the loading of EG is increased the foam density decreases as expected. It is however interesting to note that this also leads to a substantial reduction in the BET surface area, as demonstrated in Figure 8. 


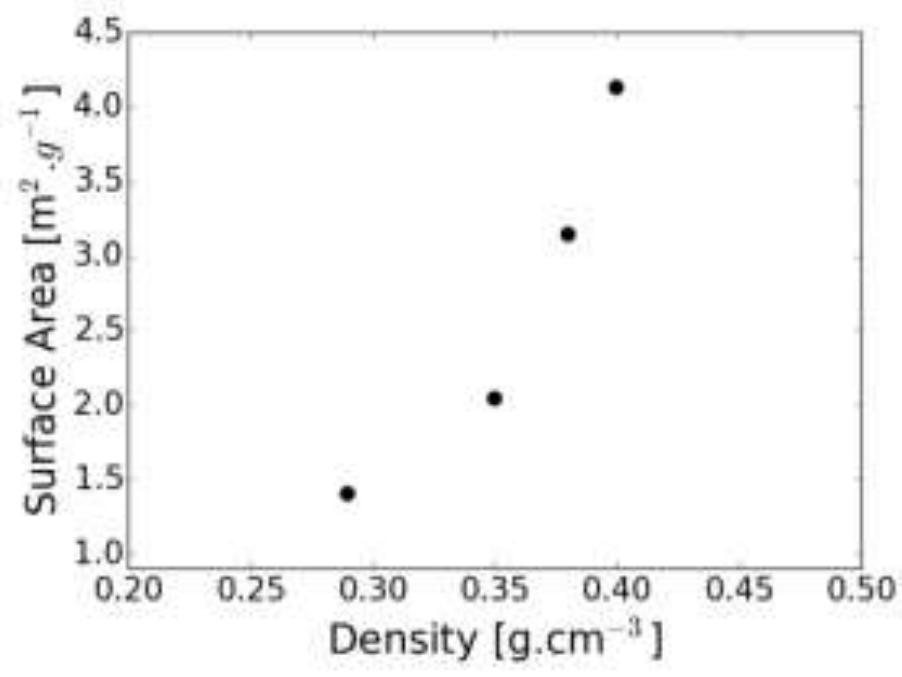

Fig. 8: Variation of foam surface area with bulk density

The reason for this reduction may be due to the fine pore structure present in the pitch derived graphite. Synthetic graphite is known to produce a fine microstructure and very small pores known as Morovski cracks [19], especially for materials with a mosaic precursor structure. As the EG loading is increased the amount of this microporous material decreases (for the same total mass) leading to an overall reduction in the surface area. It may be reasoned that this reduction in surface area would lead to a loss of performance, however as can be seen from Figure 9 this is not the case.

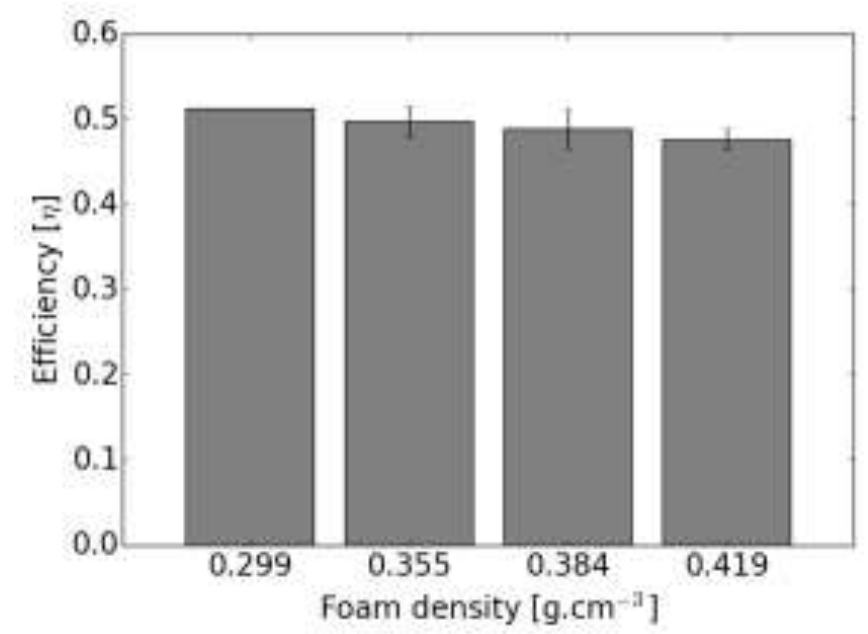

Fig. 9: Foam performance as a function of density

In fact, there is a slight reduction in the performance of the foam as the density, or equivalently surface area, increases. The reason for this is thought to be a decrease in the thermo-syphon effect whereby fluid is drawn through the foam. Due to the high 
heating rate at the top foam face, the water density is significantly reduced in this zone leading to an updraft flow. Water is drawn from the surroundings and from within the foam structure to replace the outflowing material. As the density of the foam is reduced there is less resistance to this flow and more efficient transfer of energy to the water is achieved.

This concept is in accordance with the earlier assertion that the increased surface area is caused by micro-pores since these will not contribute significantly to the heating rate. For heating the bulk movement of fluid through the macro-porosity is relevant, rather than the slow diffusion of heat out of the micro-pores. As cooler liquid is allowed to flow more freely through the foam, the average driving force or delta temperature will increase, in addition to a higher convective heat transfer coefficient due to the thinner boundary layer.

Each experiment is repeated at least five times and the standard deviations are given by the error bars in the figures. The exception is the lowest density foam which degraded significantly after the first experimental run and could not be used again. For this reason the second best performing foam with a density of $0.355 \mathrm{~g} . \mathrm{cm}^{-3}$ was chosen for subsequent analysis. In an effort to obtain the optimal foam configuration, the foam geometry was varied for the chosen foam, as detailed in Table 2.

Table 2: Foam geometry variation

\begin{tabular}{|c|c|c|}
\hline Experiment & $\begin{array}{c}\text { Thickness } \\
(\mathbf{c m})\end{array}$ & $\begin{array}{c}\text { Area (Width x Breadth) } \\
(\mathbf{c m} \times \mathbf{~ c m})\end{array}$ \\
\hline 1 & 2.9 & $2.3 \times 2.3$ \\
\hline 2 & 1.3 & $2.3 \times 2.3$ \\
\hline 3 & 0.6 & $2.3 \times 2.3$ \\
\hline 4 & 0.9 & $1 \times 1$ \\
\hline 5 & 0.9 & $1.5 \times 1.5$ \\
\hline 6 & 0.9 & $2 \times 2$ \\
\hline
\end{tabular}

The variation was done in two steps: first the foam thickness was varied while maintaining a constant upper surface area. Secondly the thickness was kept constant while the upper surface area was varied. In all cases the focal point of the lens, i.e. the 
focal size, was chosen to exactly cover the foam surface area. The reason for this choice is visible in Figure 10 below.

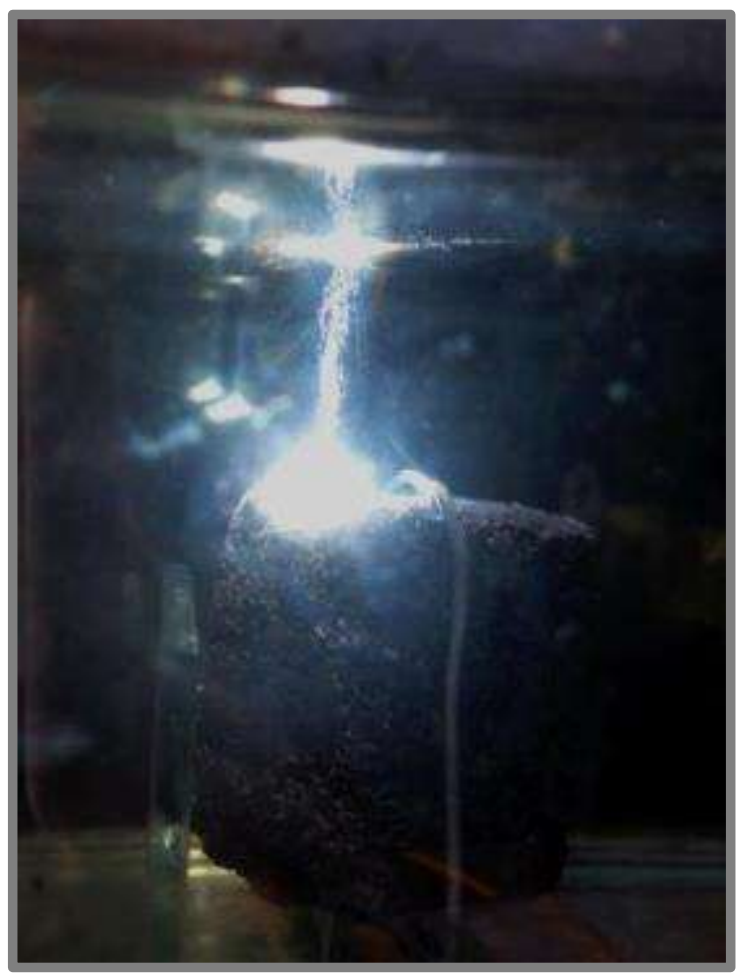

Fig. 10: Nucleate being on foam at point focus

When the focal size is reduced to its minimum value, nucleate boiling is observed. This leads to several complications, firstly the small bubbles tend to disperse the concentrated radiation, leading to increased losses. Secondly the bubbles themselves embody a significant energy flow which is not accounted for. They represent a large amount of heat in terms of the enthalpy of vaporization which is not quantified by measuring the water temperature. Thus to avoid any boiling the focal size is increased from point focus to the upper surface area of the foam. It should be noted that this does not affect the total incident radiation in any way since the size of the lens is fixed. The result of a variation in the foam thickness is demonstrated by Figure 11. 


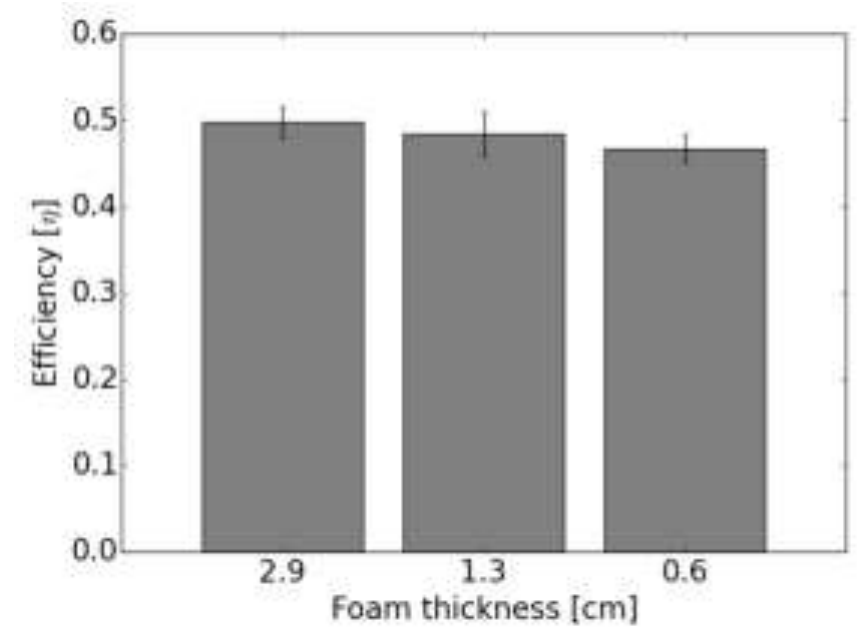

Fig. 11: Foam performance as a function of thickness

As can be seen from this figure the overall efficiency decreases as the thickness is reduced. This indicates that the entire foam surface area is being used for heat exchange. The implication is that as a result of the relatively high thermal conductivity of the foam, heat penetrates to well within the structure. This agrees with the thermo-syphon concept whereby water is being drawn into the foam from all directions and heat exchange is predominantly due to bulk convection. Thus any reduction in thickness, or that is to say the overall volume and surface area, reduces the heat transfer rate. The results of the upper surface area variation are shown below in Figure 12.

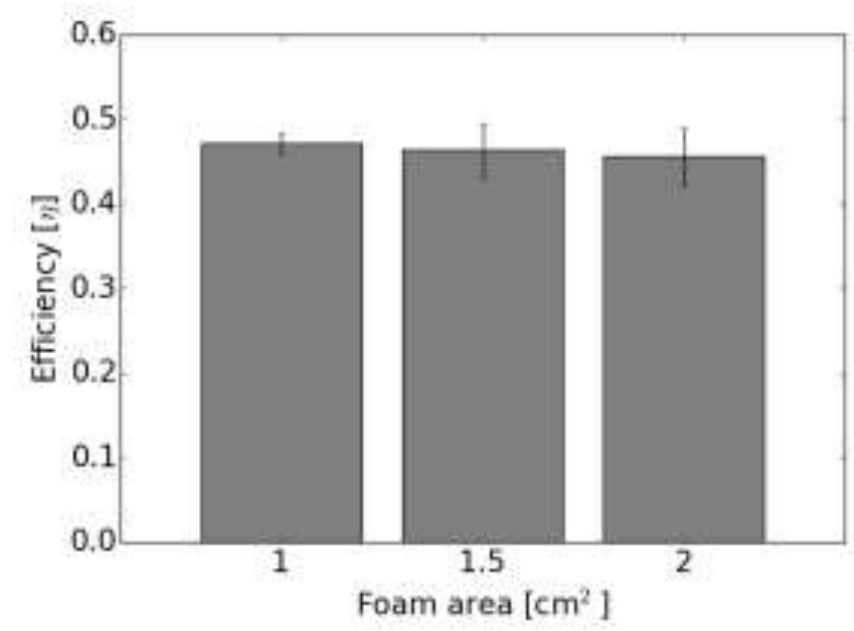

Fig. 12: Foam performance as a function of upper surface area 
The data indicates a very small reduction in the efficiency as the focal size is increased. The smaller focal size has a higher concentration ratio which results in a substantially higher foam temperature. This leads to a much higher heat transfer rate due to the increased driving force and higher thermo-syphon flow rate (due to increased buoyancy). Thus it can be seen in this case, despite a very large reduction in the foam volume ( $75 \mathrm{vol} \%)$ and associated surface area, the heat transfer rate remains more or less the same. This result indicates that for heat transfer in a flow through system, the optimal result is achieved for the highest concentration ratio. A result which also leads to a reduction in the receiver cost as its size is reduced to the minimum.

For evaluation the foam performance may be compared to that of traditional flat plate collectors. However, if this is to be done the path losses through the lens and the glass cover must be taken into account, which brings the overall system thermal efficiency to around $30 \%$. Flat plat collectors operating under similar conditions generally have an efficiency of around 35 to $40 \%$ [37, 38]. Thus it can be seen that the foams achieve a slightly lower efficiency than traditional flat plate collectors. It is believed that the graphitic nature of the foam is responsible for this. When the foam is cut its surface exhibits a very shiny texture, consistent with other types of graphite. This may reflect a portion of the light instead of absorbing it.

This investigation demonstrates that it is possible to rapidly screen different options and optimise the receiver geometry for maximum efficiency. In this case the foam with the lowest density and smallest upper surface area, or rather highest concentration ratio, should be chosen since both imply a reduction in material cost. However, care must be taken that the area is not reduced excessively, which may lead to the formation of nucleate boiling or foam breakage during cutting. In addition, the foam thickness must be chosen large enough to ensure that sufficient surface area is present for maximum heat transfer.

The situation is slightly different for the PCM since no bulk fluid flow takes place. However, since the lowest density foam still has an efficiency similar to the others it should be used. From an energy storage perspective this is the optimum choice since it will have the highest amount of PCM and hence the highest energy storage density. For a real system, the energy requirements will determine the volume of PCM which in turn will fixed the foam volume. This means that changing the concentration ratio 
will not change the receiver cost since the volume is fixed: a receiver with a large surface area will simply be thinner whilst a smaller one will be thicker. In this case other factors must be considered such as the phase change temperature. For example if phase change occurs at low temperature, concentration may not even be required. The size of the storage in combination with its required melting temperature and time will fix the design.

For the purpose of this investigation the objective is simply to (a) prove that direct capture and storage is possible and (b) give a preliminary estimate of the reduction in melting time. Thus a simple melting test was conducted from a starting temperature of $25^{\circ} \mathrm{C}$ up to a final temperature of $75^{\circ} \mathrm{C}$. In both cases the cavity size was identical, hence also the volume of material and the concentration ratio. Based on the skeletal $\left(2.0 \mathrm{~g} . \mathrm{cm}^{-3}\right)$ and bulk $\left(0.355 \mathrm{~g} . \mathrm{cm}^{-3}\right)$ densities of the foam the available volume for PCM impregnation may be calculated as $82 \%$. Which, given the myristic acid density $\left(0.99\right.$ g.cm $\left.{ }^{-3}\right)$ should result in a mass increase of $\sim 330 \%$ upon complete impregnation. This was confirmed by weighing the sample before and after. The results of the melting tests are given in Figure 13.

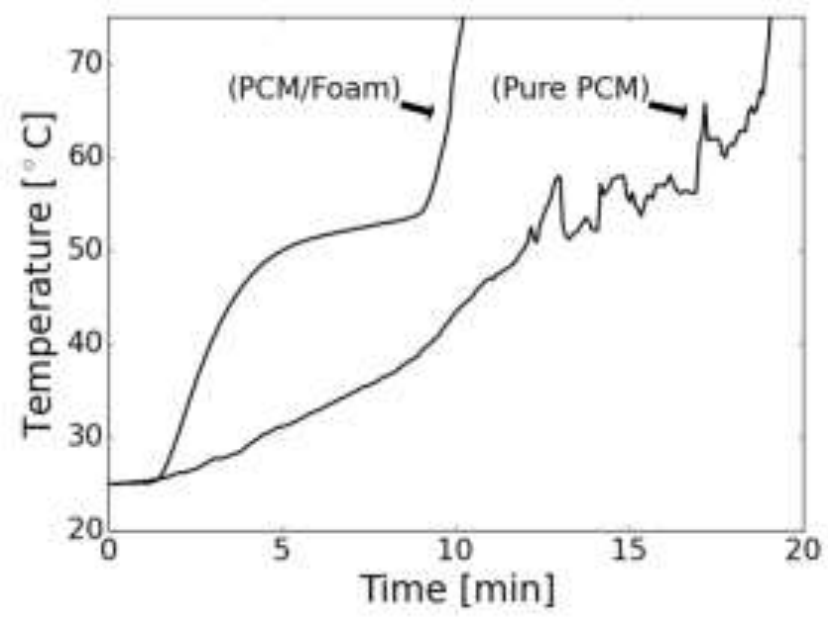

Fig. 13: Thermal performance enhancement of PCM with graphite foam

From this result it is clear that there is a significant reduction of around $46 \%$ in the melting time of the composite compared to the pure PCM. However, several additional factors should be borne in mind. Firstly the melting of the PCM is greatly assisted by natural convection. This would not be the case for freezing, thus during this transition the performance enhancement of the foam would be significantly larger. Secondly, the nature of the concentrator creates a narrowing point of focus 
which tends to melt the core of the PCM whilst leaving the walls solid. This is related to another issue with the use of PCMs that is seldom mentioned but is clearly demonstrated by Figure 13: incongruent melting.

When the PCM solidifies its density is increased, leading to the formation of cavities within the structure. This results in inconsistent melting and the noise, or erratic behaviour, observed during the melting of the pure PCM in Figure 13. The behaviour of the foam composite on the other hand is very close to ideal, a clear plateau is visible during the phase transition itself, with an almost constant temperature. This is stated as one of the key advantages of using PCM energy stores, i.e. heat release at constant temperature. However this may not be practically achievable using the PCM alone but can be realized with the foam composite. From the DSC results the energy density of the pure PCM (during phase transition) was measured at $202.7 \mathrm{MJ} . \mathrm{m}^{-3}$. Given the volumetric loading of the composite this would be reduced to $166.8 \mathrm{MJ} . \mathrm{m}^{-3}$ compared to the pure PCM.

\section{Conclusions and recommendations}

Solar collectors and thermal energy storage components are two key subsystems in most solar thermal applications. Graphite foams have several properties which make them ideal for use in these applications, including high thermal conductivity, low porosity, high surface area and low thermal expansion. Testing materials for concentrated solar applications can be costly and achieving representative results of real operating conditions is difficult. For this reason a simple device has been developed to track and reflect sunlight onto a Fresnel lens based concentrator. The device uses only low cost materials and is capable of concentration ratios in excess of 1,000 suns. This system was used as the platform for assessing different graphitic foams for concentrated solar applications.

Traditionally graphite foams are produced from scarce and expensive mesophase pitch. Instead graphite foams of varying composition and density were prepared using a locally produced pitch material and expandable graphite. These foams demonstrate a high degree of graphitization but exhibit a fine mosaic texture in the final product. A small oxidative treatment ( $6 \%$ mass loss) was necessary to fully open the foam pores. The efficiency of the foams in transferring incident solar energy was tested using the concentrator platform and a water calorimeter. A reduction in density resulted in a 
large decrease in the measured foam surface area. Despite this fact, an increase in efficiency was observed. The reason for this is believed to be a thermo-syphon effect whereby water is pulled through the foam structure. A decrease in density results in lower resistance to flow and consequently a higher heat transfer rate.

The geometric configuration of the foam was also considered by varying the foam thickness and exposed surface area. In this way the concentration ratio of the incident radiation was varied. The results indicate that due to the good thermal conductivity of the foam heat penetrates to well within the structure and a decrease in thickness resulted in a decreased efficiency. This would be mainly due to the reduction in the area for heat transfer. Remarkably as the size of the exposed surface is decreased and hence the concentration ratio increased, the efficiency remains the same. This demonstrated that the receiver size can be reduced by $75 \%$ whilst still yielding the same efficiency. However, as the focal point is reduced further, a point is reached where nucleate boiling is observed. Whilst detrimental to the current investigation, this may be useful for solar distillation applications.

Overall foam efficiency was somewhat lower $(\sim 30 \%)$ than what is achievable with conventional flat plate collectors $(\sim 38 \%)$ under similar conditions, this may be due to the reflective nature of graphite. Future work will investigate the incorporation of small amounts of amorphous carbon such as carbon black into the precursor mixture to increase light absorption. In addition, focus will be placed on improving the foam microstructure. By increasing the mesophase domain sizes and their alignment within the foam it is believed that increased thermal conductivity will lead to better performance. This can be achieved by varying the milled pitch particle size and possibly the foaming temperature.

This procedure enabled a rapid screening and comparison of the different foam materials and configurations. The most attractive material was then selected for testing the thermal performance enhancement of a PCM. Based on the results the sample with the lowest density was used. This also maximizes the energy storage density as it allows maximum PCM loading. To assess the performance the time required for complete melting of the PCM was compared to that of the pure PCM. It was found that the foam is capable of reducing the melting time by $46 \%$ whilst only reducing the energy storage density by $18 \%$. In addition it was found that the foam composite resulted in far more ideal phase transition behaviour. The reason is that the 
incongruent melting encountered in the pure PCM is negated. In this way heat transfer from the energy storage media at a constant temperature can now be achieved in practice.

In conclusion, the composite has the potential to effectively capture, store and discharge thermal energy, at a constant temperature, without any additional requirements. This work demonstrates a novel approach to energy storage is possible which can increase the availability of renewable energy resources.

\section{Acknowledgements}

This work is based on the research supported by the South African Research Chairs Initiative of the Department of Science and Technology and National Research Foundation of South Africa (Grant No 97994). Any opinion, finding and conclusion or recommendation expressed in this material is that of the author(s) and the NRF does not accept any liability in this regard.

\section{References}

[1] Lise W, van der Laan J. Investment needs for climate change adaptation measures of electricity power plants in the EU. Energy Sustain Dev, 2015;28:10-20.

[2] Zhoa X, Du D. Forecasting carbon dioxide emissions. J Environ Manage, 2015;160:39-44.

[3] European Commission. Concentrating solar power - From research to implementation. Published in Luxembourg by Office for Official Publications of the European Communities; 2007. ISBN 978-92-79-05355-9. ec.europa.eu.

[4] Sargent \& Lundy LLC Consulting Group. Assessment of parabolic trough and power tower solar technology cost and performance forecasts. Report NREL/SR550-34440, NREL (National Renewable Energy Laboratory), Golden, CO,USA; 2003.

[5] Gevrek ZE, Uyduranoglu A. Public preferences for carbon tax attributes. Ecol Econ, 2015;118:187-97.

[6] Hertwich EG, Peters GP. Carbon Footprint of Nations: A Global, Trade-Linked Analysis. Environ Sci Technol, 2009; 43:6414-20. 
[7] Wu Z, Lui Z, Parvez K, Feng X, Müllen K. Ultrathin Printable Graphene Supercapacitors with AC Line-Filtering Performance. Adv Mater, 2015;27:366975.

[8] Konios D, Petridis C, Kakavelakis G, Sygletou M, Savva K, Stratakis E, Kymakis E. Reduced Graphene Oxide Micromesh Electrodes for Large Area, Flexible, Organic Photovoltaic Devices. Adv Func Mater, 2015;25:2213-21.

[9] Tian Y, Zhao CY. A review of solar collectors and thermal energy storage in solar thermal applications. Appl Energy, 2013;104:538-53.

[10] Baharoon DA, Rahman HA, Omar WZW, Fadhl SO. Historical development of concentrating solar power technologies to generate clean electricity efficiently - A review. Ren Sust Energy Rev, 2015;41:996-1027.

[11] Ho CK, Iverson BD. Review of high-temperature central receiver designs for concentrating solar power. Ren Sust Energy Rev, 2014;29:835-46.

[12] Behar O, Khellaf A, Mohammedi K. A review of studies on central receiver solar thermal power plants. Ren Sust Energy Rev, 2013;23:12-39.

[13] Alonso E, Romero M. Review of experimental investigation on directly irradiated particles solar reactors. Ren Sust Energy Rev, 2015;41:53-67.

[14] Krueger KR, Davidson JH, Lipinski W. Design of a New 45 kWe High-Flux Solar Simulator for High-Temperature Solar Thermal and Thermochemical Research. J Sol Energy Eng, 2011;133:0110131-8.

[15] Alxneit I, Schmit H. Spectral Characterization of PSI's High-Flux Solar Simulator. Research. J Sol Energy Eng, 2012;134:0110131-5.

[16] Petrasch J, Coray P, Meier A, Brack M, Haberling P, Wuillemin D, Steinfeld A. A Novel 50 kW11,000 Suns High-Flux Solar Simulator Based on an Array of Xenon Arc Lamps. ASME J Sol Energy, 2007;129:405-11.

[17] Xie WT, Dai YJ, Wang RZ, Sumathy K. Concentrated solar energy applications using Fresnel lenses: A review. Ren Sust Energy Rev, 2011;15:2588-606.

[18] Sani E, Mercatelli L, Francini F, Sans J-L, Sciti D. Ultra-refractory ceramics for high-temperature solar absorbers. Scr Mater, 2011;65:775-8.

[19] Pierson HO. Handbook of Carbon, Graphite, Diamond and Fullerenes. Properties, Processing and Applications. 1993; Noyes Publications, New Jersey, USA. 
[20] Avila-Marin AL. Volumetric receivers in Solar Thermal Power Plants with Central Receiver System technology: A review. Sol Ener, 2011;85:891-910.

[21] Yadav A, Kumar R, Bhatia G, Verma GL. Development of mesophase pitch derived high thermal conductivity graphite foam using a template method. Carbon, 2011;49:3622-30.

[22] Klett J, Hardy R, Romine E, Walls C, Burchell T. High-thermal conductivity, mesophase-pitch-derived carbon foams: effect of precursor on structure and properties. Carbon, 2000;38:953-73.

[23] Klett JW, McMillan AD, Gallego NC, Burchell TD, Walls CA. Effects of heat treatment conditions on the thermal properties of mesophase pitch-derived graphitic foams. Carbon, 2004;42:1849-52.

[24] Gaies D, Faber KT. Thermal properties of pitch-derived graphite foam. Carbon 2002;40(7):1137-40.

[25] Anderson HJ, Anderson DP, Kearns KM. Microcellular pitch based carbon foams blown with helium gas. p. 756-62.

[26] Chen C, Kennel EB, Stiller AH, Stansberry PG, Zondlo JW. Carbon foam derived from various precursors. Carbon 2006;44(8):1535-43.

[27] Focke WW, Badenhorst H, Ramjee S, Kruger HJ, van Schalkwyk R, Rand B. Graphite foam from pitch and expandable graphite. Carbon, 2014;73:41-50.

[28] Becker M, Fend T, Hoffschmidt B, Pitz-Paal R, Reutter O, Stamatov V, Steven M, Trimis D. Theoretical and numerical investigation of flow stability in porous materials applied as volumetric solar receivers. Sol Energy, 2006;80:1241-8.

[29] Kuravi S, Trahan J, Goswami DY, Rahman MM, Stefanakos EK, Thermal energy storage technologies and systems for concentrating solar power plants. Prog Energy Combust Sci, 2013;39: 285-319.

[30] Zeng J, Zheng S, Yu S, Zhu F, Gan J, Zhu L, et al. Preparation and thermal properties of palmitic acid/polyaniline/exfoliated graphite nanoplatelets formstable phase change materials. Appl Energy, 2014;115:603-9.

[31] Badenhorst H. Performance comparison of three models for thermal property determination from experimental phase change data. Thermochim Acta, 2015;616:69-78 
[32] Lafdi K, Mesalhy O, Elgafy A. Graphite foams infiltrated with phase change materials as alternative materials for space and terrestrial thermal energy storage applications. Carbon, 2008;46:159-68.

[33] Zhong Y, Guo Q, Li S, Shi J, Lui L. Heat transfer enhancement of paraffin wax using graphite foam for thermal energy storage. Sol Energy Mater Sol Cells, 2010;94:1011-4.

[34] Yadav A, Kumar R, Bhatia G, Verma GL. Development of mesophase pitch derived high thermal conductivity graphite foam using a template method. Carbon 2011;49(11):3622-30.

[35] Gallego NC, Klett JW. Carbon foams for thermal management. Carbon 2003;41(7):1461-6.

[36] Zalba B, Marín JM, Cabeza LF, Mehling H. Review on thermal energy storage with phase change: materials, heat transfer analysis and applications. Appl. Therm. Eng. 2003;23:251-83.

[37] Pluta Z. Evacuated tubular or classical flat plate solar collectors? J. Power Technol. 2011;91:158-64.

[38] Ehrlich R. Renewable energy: a first course. 2013; CRC Press, Boa Raton, USA. 